\title{
Approach to Implementation of Local Navigation of Mobile Robotic Systems in Agriculture with the Aid of Radio Modules
}

\author{
Roman Iakovlev and Anton Saveliev
}

\begin{abstract}
In this paper an approach is presented, enabling to solve the problem of local navigation of mobile robotic platforms (MRP), based on utilization of wireless networks with mesh topology. Establishment of wireless networks was ensured, based on the set of radio modules, mounted on unmanned aerial vehicles (UAV), comprising a swarm. This paper presents a developed algorithm for establishment of such wireless networks, aided by LoRa-technology, as well as an algorithm for MRP localization, based on analysis of signal level, where the incoming signals are fed from MRP group radio modules to radio modules of wireless data transfer network. An algorithmic model is given for task distribution among UAV and to implement navigational capabilities of MRP swarm. In some experiments descending dependencies of absolute error value, pertinent to MRP, from the number of $\mathrm{UAV}$ in action were revealed, as well as of averaged deflection value of MRP positions in motion along their paths from the number of $U A V$ in action. Thereby the averaged value of MRP localization error, depending on the number of UAV in action, was from 8.14 to $17.13 \mathrm{~m}$, and the averaged value of MRP position deflection - from 16.38 to $57.12 \mathrm{~m}$, respectively.
\end{abstract}

Keywords - robot navigation, LoRa, radio communication, mesh topology, mesh networks.

\section{INTRODUCTION}

$\mathrm{C}$ urrently agricultural robots are employed to solve a wide range of problems: field activities, crop harvesting, livestock breeding, monitoring of agricultural areas, etc. [1]. Utilization of such automated robotic vehicles in open agricultural areas is complicated by high variability of the environment and weather conditions, and often requires establishment of a robust wireless connection among robotic vehicles, due to vastness of the terrains to be served [2]. Long distances and intermittent GPS connections in agricultural areas far from big cities, significantly complicate the utilization of GPS-based navigational methods [3], [4] and extremely negatively

Paper received July 14, 2020; revised October 17, 2020; accepted Oktobre 21, 2020. Date of publication December 25, 2020. The associate editor coordinating the review of this manuscript and approving it for publication was Prof. Aleksandar Nešković.

Corresponding author Roman Iakovlev is with the St. Petersburg Federal Research Center of the Russian Academy of Sciences (SPC RAS), St. Petersburg Institute for Informatics and Automation of the Russian Academy of Sciences, St. Petersburg, Russia; (phone: +7(812)328-3411, e mail: iakovlev.r@mail.ru).

Anton Saveliev is with the St. Petersburg Federal Research Center of the Russian Academy of Sciences (SPC RAS), St. Petersburg Institute for Informatics and Automation of the Russian Academy of Sciences, St. Petersburg, Russia; (phone: $+7(812) 328-3411$, e mail: saveliev.ais@yandex.ru). influence their performance quality. As an alternative solution to ensure the navigation of agricultural robotic vehicles, the technologies of wireless data transfer may be employed, based on radio modules [5]. Integration of radio modules into a mesh network provides for wide-range wireless communication, which is enough fault-tolerant, and the whole system remains operational in the case of failure of individual network nodes. Existing applications of wireless mesh networks are presented in papers [6]-[9].

Generally, all the existing methods and approaches show decent performance quality, but do not assume modular network establishment, where the network units could maintain bidirectional radio communication over a long distance. Besides, these solutions rely on the possibility to establish the wireless data transfer network directly based on those agents, whose correct positioning should be established. Simultaneously, the specifics of the agricultural domain imply greater spatial sparsity of the robotic devices in use. Accordingly, within the operational radius of the radio module, mounted on a certain robotic device, no other robotic device may be found, what precludes wireless data transfer and, consequently, renders impossible the implementation of the navigational methods, mentioned above. In this paper, to ensure the local navigation of MRP set $M$, tasked with agricultural activities in bounded outdoor agricultural areas, UAV set $(A)$ utilization is proposed, where specialized radio modules are mounted on aerial vehicles. Using radio modules for establishment of an ad-hoc mesh wireless network, based on an UAV group, provides for implementation of a wireless navigational system, which remains functional without bearing on external positioning systems. This paper presents an approach, ensuring robotic navigation in outdoor agricultural areas, as well models and algorithms, pertinent to this approach.

\section{OVERVIEW OF THE DEVELOPED APPROACH FOR IMPLEMENTATION OF LOCAL NAVIGATION}

The proposed approach to the implementation of local MRP navigation in outdoor agricultural areas in the context of significant mutual sparsity of robotic agents requires the following problems to be solved: establishment of a wireless modular mesh networks for data transfer, where individual data transfer modules are mounted on UAV; development of a respective localization algorithm for a MRP, connected to such network; development of a task distribution model to include different UAV in the process of MRP group navigation. 
Consider the developed algorithm for wireless data transfer, based on radio modules, which are mounted on group UAV (Fig. 1). This algorithm enables one to determine target coordinates of an UAV swarm to maintain the required data transfer speed in the network being deployed. In this research, LoRa [10] is used as a base technology for establishment of a wireless data transfer network, because it supports a wide range of operational frequencies, broadband communication line, secure data transfer protocol. The radio modules, required for it, are relatively cheap and enable a seamless connection between modules at distances up to $3 \mathrm{~km}$.

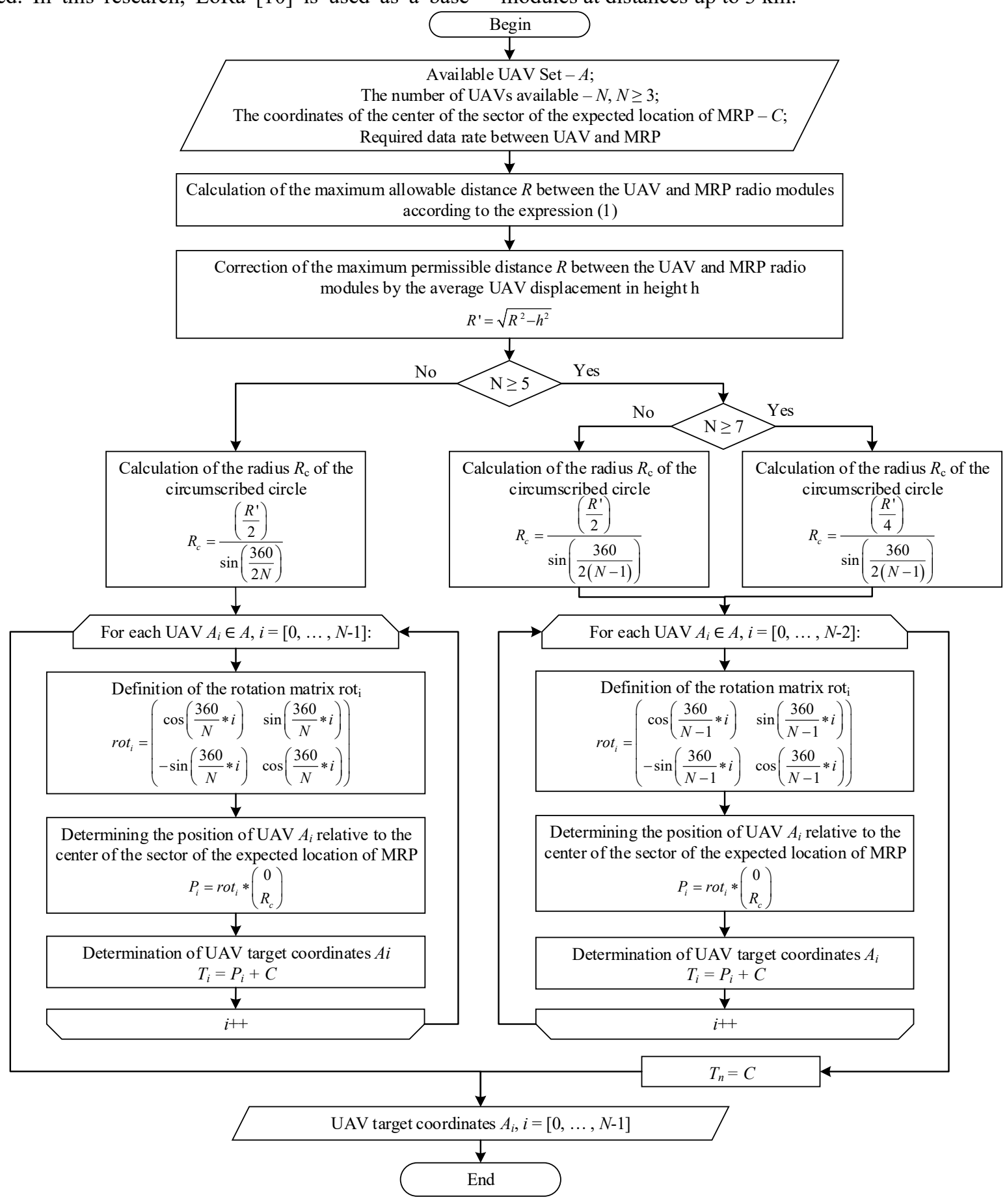

Fig. 1. Algorithm for establishment of a mesh network, based on group of UAV' radio modules.

This algorithm receives as input the current coordinates of UAV, the number of UAV in the actual set, central coordinates of the segment, where the MRP is expected to be found, as well as the required speed of data transfer between the radio modules, mounted on groups UAV and MRP, as well as other parameters of communication. To ensure the required data transmission speed among network nodes, in the first stage of the algorithm the calculation of maximum allowable distance $R$ between radio modules is performed (1):

$$
R=\frac{3 \cdot 10^{8}}{4 \pi F} \cdot 10 \frac{P_{T}+G_{T}-L_{T}+G_{R}-L_{R}-|V| d B-P_{R}}{20} \cdot \partial,
$$

where $R$ - target communication distance, $\mathrm{m} ; F$ frequency, $\mathrm{Hz} ; P_{T}-$ transmitter power, $\mathrm{dBm} ; G_{T}-$ transmitter antenna gain, $\mathrm{dB} ; L_{T}-$ losses in wire, leading to transmitter antenna, $\mathrm{dB} ; G_{R}-$ receiver antenna gain, $\mathrm{dB}$; 
$L_{R}-$ losses in wire, leading to receiver antenna, $\mathrm{dB} ; P_{R}-$ radio receiver sensitivity, $\mathrm{dBm} ;|V| d B$ - attenuation factor, which accounts for additional loss, caused by influence of the Earth, vegetation, atmosphere and other components, $\mathrm{dB}, \partial$ - adjustment ratio, equal to 0.85 [11].

Within the framework of this research, the signal attenuation coefficient was estimated through a number of preliminary experiments in the area, where the solution was tested: at known positions of the transmitters, similar to those installed on groups UAV and MRP, a series of measurements of the received signal level was carried out at different times of day and in different weather conditions. These measurements were carried out at different parts of the surveyed area in the vicinity of potential routes of MRP. Thus, a matrix of estimated values of the attenuation factor for a given territory was obtained. These estimated values were used in the expression (1). Specific values were taken as values corresponding to the center of the sector, where the MRP is expected to be located.

Because the signal level changes, together with flight altitude of the UAV, when UAV group is utilized in dynamical network deployment, it is necessary to consider the vertical shift for onboard radio modules relative to landbased radio modules. To do this, in the next step the respective distance adjustment $R$ is performed, upon which the corrected distance $R^{\prime}$ is determined. Further steps depend on the number of UAV, employed in the establishment of data transfer network and are intended to ensure uniform distribution of UAV group along the circumference with a radius $R_{c}$, drawn around some regular polygon, whose center point corresponds to the origin of the segment, where the MRP is expected to be (Fig. 1). Radius $R_{c}$ of the circumference, drawn this way, is determined according to the following expression:

$$
\left\{\begin{array}{c}
R_{c}=\frac{\alpha}{2 \sin (360 / 2 N)}, N<5 \\
R_{c}=\frac{\alpha}{2 \sin (360 / 2(N-1))}, 5 \leq N \leq 7 \\
R_{c}=\frac{\alpha}{4 \sin (360 / 2(N-1))}, N \geq 7
\end{array}\right.
$$

where $N$ - the number of UAV in action, $\alpha-$ the side of the polygon, equal to $R$ '.

Using the above expression to determine the radius $R_{c}$ allows the following conditions to be satisfied over the entire considered range of $N$ (will be discussed below): a) the distance between the target positions of any two neighbor UAV is less than or equal to $R^{\prime} ; \mathrm{b}$ ) any UAV can transmit data to any other UAV from this set, via at most one signal hop; c) any MRP, being in this circumference, is within the coverage of at least three UAVs simultaneously.

At the final stage of the algorithm, the positions of UAV relative to the center of the segment, where the MRP is supposed to be, are translated into spatial coordinates. The output of the proposed algorithm contains target coordinates for the supplied group of UAVs, based on which the wireless data transfer network is established, ensuring the required level of data transfer speed. Further consider the developed algorithm for localization of MRP, being within the coverage of such wireless network.

Search for a terrain segment, corresponding to the MRP position, can be performed upon the analysis of signal levels, where the signal in question is fed from the radio module of the current system to the radio modules of other units of the wireless data transfer system. As with a distance increase from transmitter to receiver, the signal level drops, a circumference $C_{i}$ with radius $R_{i}$ can be outlined around each receiver, which detects a signal from a known source; here the $R_{i}$ corresponds to the calculated distance between source and receiver by a certain signal level. These distances can be found using expression (1), provided that the transmitter power and signal level at receiver are known. In this case the terrain segment, corresponding to the MRP position, can be presented as a circle with area $S_{r}$ and center at $C_{0}$, corresponding to the center of intersection of circumferences $C_{i}(i=[1, \ldots, N])$. The localization algorithm includes the following steps:

- sending an interaction initiation signal from UAV to MRP;

- sending a response signal from MRP;

- signal receiving by beacons on a UAV;

- data collection at each UAV: UAV location coordinates, intensity of the received signal;

- sending data by each of the UAV to the MRP;

- MRP-based calculation of the distances from each beacon installed on the UAV to the signal source according to expression (1);

- determining the estimated location of the object based on the intersection of circles according to [12];

- representation of the estimated location of the object as a polygonal shape with the number of vertices $N$;

- divide the resulting polygon into $N$ triangles, whose vertices match to the vertices of the polygon;

- calculation of geometric centers for each of $N$ triangles and the respective area values for them;

- revealing the center point of the intersection of all circles $C_{o}$ according to expression (3);

- sending object location data from MRP to UAV.

To simplify calculations in terms of the developed algorithm, the circumference intersection area $C_{i}$ is presented as a circle, whose center point corresponds to the center of this segment, and whose area is equal to the area of this segment. As the obtained common area at the intersection of the circumferences is the shape, formed by arcs, to simplify the detection of its center (geometrical center is the arithmetic mean of the positions of all points within this shape), the obtained intersection area is represented as a polygon [12]. This polygon is divided into triangles, whose number is equal to the number of polygon sides; then the area and centroid are calculated for each of the obtained triangles. As the resulting estimate of the MRP position in this case the weighted estimate $C_{0}=\left(x_{0}, y_{0}\right)$ is used, which characterizes the position of center point of the intersection area between the circumferences $C_{i}$, established according to the following expression (3):

$$
x_{0}=\frac{\sum_{i=1}^{N} x_{i} * S_{i}}{\sum_{i=1}^{N} S_{i}}, \quad y_{0}=\frac{\sum_{i=1}^{N} y_{i} * S_{i}}{\sum_{i=1}^{N} S_{i}},
$$


where $N$ - number of triangles, $\left(x_{i}, y_{i}\right)$ - coordinates of the center point of the $i$-th triangle, $S_{i}$ - area of the $i$-th triangle.

The developed algorithm allows localizing the robotic platform using radio beacons, mounted on a group of UAVs, even if it is outside of direct visibility of technical vision systems, embedded in the devices under consideration.

Further consider the developed model for task distribution among UAVs during the navigation of MRP group. It is supposed within this research, that the solution for MRP navigation, established according to the approach, outlined above, is deployed in some agricultural area, where the specified group of MRP is utilized. During motion, group of MRP deflect from the traced paths due to

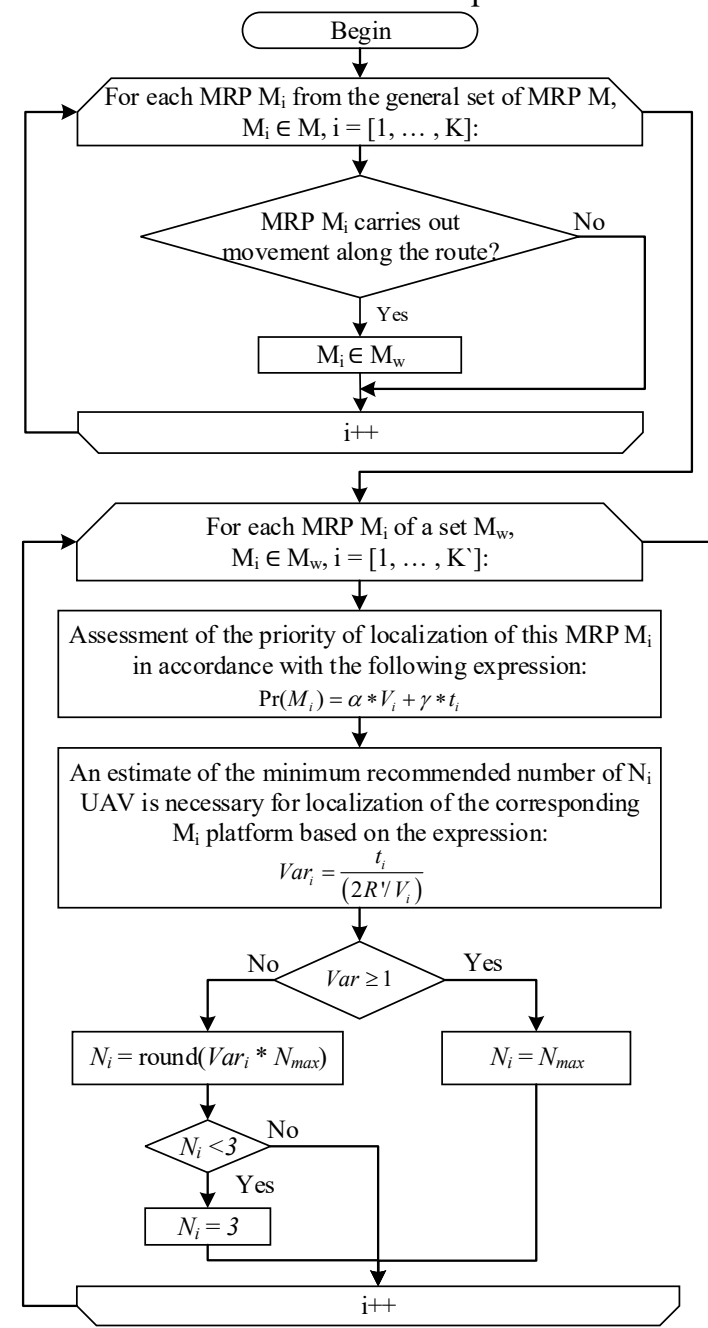

Fig. 2. Algorithmic model of task distribution among group of UAVs to ensure MRP navigation.

The proposed algorithmic model assumes execution of the following main operations in the loop:

1. estimation of priority for certain MRP localization tasks;

2. estimation of necessary resource amount to complete the tasks;

3. distribution of each UAV across the current tasks.

In the first stage of the algorithm identification of those MRP is performed, which should be localized with the aid of UAV group. To do this, from the general MRP set a subset Mw is extracted, to which any MRPs are added, which are currently following the traced paths. Then for every MRP $M_{i}$ from the Mw subset the priority of accumulated error of the embedded positioning systems. The developed model enables one to look up the coordinates of actual MRP position using a group of UAVs for further adjustment of MRP paths.

During MRP motion along paths with a certain time interval $t$ a set of tasks is established, concerning the localization of MRP. Here it is important to note, that by decreasing the rate of MRP localization checks the accumulated error grows, hence, the areas, where the MRP is supposed to be, will also increase in size, what requires to put in action more UAVs to solve every particular task. To keep track of the distribution of available UAV across current localization tasks, a respective algorithmic model was developed, presented further, in Fig. 2.

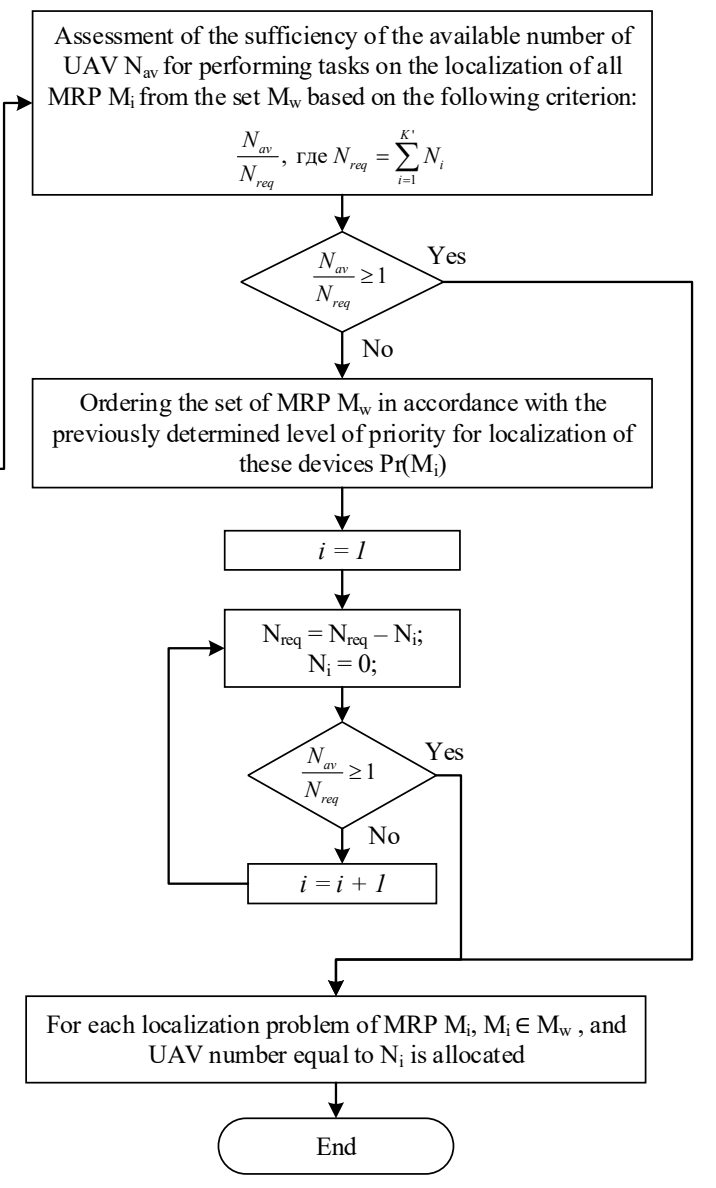

localization of the certain platform is estimated, according to the following expression:

$$
\operatorname{Pr}\left(M_{i}\right)=\left(\alpha * V_{i}\right) *\left(\gamma * t_{i}\right),
$$

where $V_{i}$-calculated motion velocity of MRP $M_{i}$ along the route $(\mathrm{km} / \mathrm{h}), t_{i}$ - time elapsed from the moment, when this MRP was localized most recently using UAV, $\alpha$ and $\gamma-$ empirically specified weight coefficients for factors $V$ and $t$ respectively.

Further, the amount of resources is estimated, necessary to complete the current localization tasks. Concerning every MRP $M_{i}$ from the subset Mw the minimum recommended number of UAV $N_{i}$ is determined, necessary to localize the 
respective platform. This estimate is based on the following expression:

$$
\operatorname{Var}_{i}=\frac{t_{i}}{\left(2 R^{\prime} / V_{i}\right)},
$$

where $R^{\prime}$ - adjusted threshold distance between radio modules, at which a guaranteed seamless connection is maintained; the approach to the calculation of this value is presented above within the algorithm in flowchart 1 . This expression indirectly characterizes the ratio of potential accumulated value of deflection of $M_{i}$ platform from the motion path to the double threshold distance $R$ '. A minimal recommended UAV number as such, necessary to localize the platform $M_{i}$, is defined by the following expression:

$$
\left\{\begin{array}{ll}
N_{i}=\operatorname{round}\left(\operatorname{Var}_{i} * N_{\text {max }}\right), & \text { Var }<1 \\
N_{i}=N_{\text {max }}, & \text { Var } \geq 1
\end{array},\right.
$$

where $N_{\max }$ - maximum number of UAVs, available for MRP localization - is chosen from the range $[10, \ldots, 12]$ (will be discussed in section III).

At the final stage of the algorithm it is determined, if the available resources suffice to perform all the tasks, concerning MRP localization and further distribution of the available group of UAVs across the specific tasks. The estimation of resource sufficiency is performed via comparing of necessary UAV number $N_{\text {req }}$ with the actually available number $N_{a v}$. The respective value of resource sufficiency $A_{v}$ is defined as the ratio of $N_{a v}$ to $N_{\text {req. }}$ In the case if $A_{v} \geq 1$ for every task of MRP localization $L_{i}\left(L_{i} \rightarrow M_{i}\right)$, the previously defined number of UAV $N_{i}$ is dedicated for the task. Otherwise, according to the accepted workflow strategy in the case of resource insufficiency, a certain number of the tasks with the lowest priority levels are discarded. Therefore, this strategy of UAV distribution is intended to maximize the MRP localization accuracy via utilization of the recommended number of UAV $N_{i}$ to solve every of the remaining MRP localization tasks $L_{i}$. Further proceed to the experimental estimation of the developed approach to implementation of local MRP navigation, which leverages radio modules, mounted on UAV.

\section{EXPERIMENTAL ESTIMATION OF THE DEVELOPED APPROACH TO IMPLEMENTATION OF LOCAL MRP NAVIGATION}

In the context of this research some experiments were performed, aimed to estimate the quality of navigational system of the MRP, where this system was implemented on premise of the approach, presented above. In the experiments 20 unmanned aerial vehicles Ryze Tech Tello [13] were utilized, additionally enhanced by radio modules Heltec ESP-32 LoRa, mounted on them. As ground-based vehicles for outdoor areas $3 \mathrm{MRP}$, described in [14], [15], were utilized. For the experiments, a plot of agricultural land of $9 \mathrm{~km}^{2}$ was used, the surface of this territory was predominantly flat, while there were areas without vegetation, with cereal crops, as well as neglected grass plots with sparse shrubbery.

The first of the performed experiments was intended to estimate the accuracy of MRP localization algorithm, depending on the number of UAVs, employed in localization tasks. Within this experiment some suites of tests were performed, 100 tries each, where the number of UAVs in action varied from 3 to 12 . Thereby the adjusted threshold distance $R$ ' between the radio modules, mounted on a group of UAVs, where seamless radio communication could be ensured, was about $1 \mathrm{~km}$. As a metric, characterizing the MRP localization accuracy, the absolute localization error ( $\mathrm{m}$ ) was used. This error is determined, comparing data on MRP position, obtained via the developed method, with the reference data set, obtained with GIS Satellite software [16], enabling to retrieve object positions from the orthophoto map with accuracy within several centimeters. The values of averaged absolute localization error, depending on the number of UAV in use, obtained within the performed experiments, are given in Fig. 3.

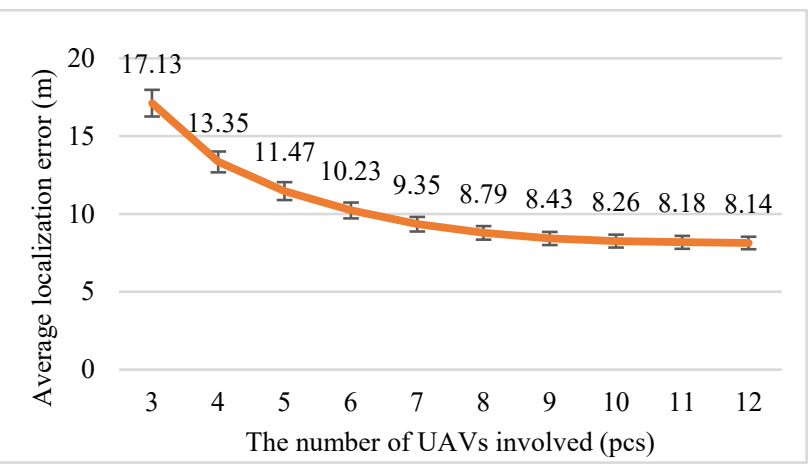

Fig. 3. Values of averaged absolute localization error, depending on the number of UAV in use.

As evident from the chart, with an initial increase of UAV number the localization accuracy improves; particularly, using 6 UAVs instead of a minimum number of 3 ensures accuracy boost of $67.4 \%$. But, by a further increase in UAV number, the localization accuracy boost reduces significantly; so, when using $12 \mathrm{UAV}$ instead of 6 , relative accuracy boost makes up only $25.6 \%$ on the average. A further increase in the number of UAVs involved weakly affects the accuracy of localization; an accuracy boost with an increase in the number of UAV from 10 to 12 on the average is less than $1.5 \%$. It is noteworthy, though, that, increasing the number of UAVs in use according to the algorithm of mesh network establishment (Fig. 1), the size of the segment also increases, within which the MRP is possible to be localized, hence, greater deflections of MRP from the calculated path become possible to be processed. Particularly, in the settings with 3, 6 and 12 UAVs in action, the MRP localization area is approximately $1.04,2.21$ and $2.49 \mathrm{~km}^{2}$, respectively. Hence, increasing the number of UAVs in use, we can not only increase the MRP localization accuracy, but also extend the area of potential search zone. Based on the obtained experimental results, the optimal range for the maximum number of UAVs involved in the process of localization of a single MRP $\left(N_{\max }\right)$ was assumed to be $[10 \ldots 12]$.

The second experiment was aimed to estimate the MRP navigation accuracy, based on the proposed models and algorithms Within this experiment some suites of tests were performed, 30 tries each, where the number of available 
UAV varied from 6 to 20, and the number of MRP end route was 3. Thereby, in each test the accumulated absolute deflection of each MRP from the traced path. Upon averaging the obtained values over the set of MRP and over the set of tests, the curve for this value was established, showing its dependency on the number of available UAVs. The resulting dependency is shown in Fig. 4.

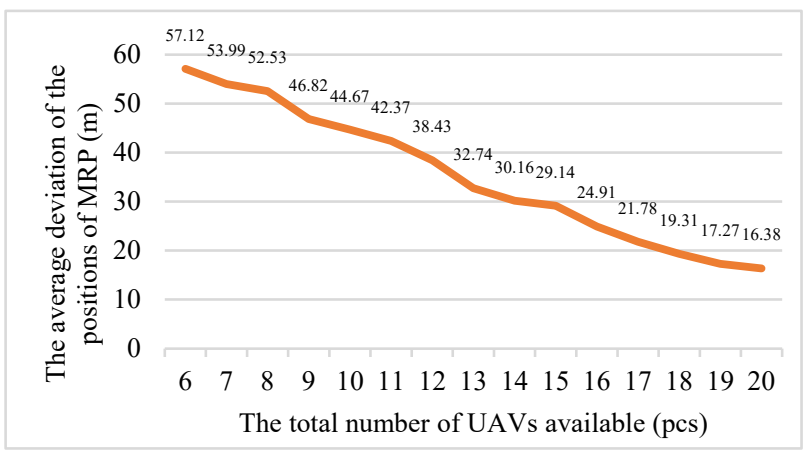

Fig. 4. The dependency of the averaged value of the accumulated absolute MRP deflection on the total number of available UAVs.

The graph, presented above, shows a steady decrease of the averaged value of MRP deflection with the increase of total number of available UAVs, that may be employed in localization problem solving. This dependency is explained as follows: with the increase in the total number of available UAVs, whereby the number of active MRP remains unchanged, the average rate of localization checks for each individual MRP increases. This, in turn, reduces the accumulated error in MRP positioning, because the time intervals between localization checks for each MRP. Therefore, the experimental results lead us to conclude, that the developed navigation system remains functional by different numbers of available UAVs, what allows in every particular instance to find a tradeoff between MRP navigation accuracy and the number of UAVs, required for the system to remain functional.

\section{CONCLUSION}

In remote agricultural areas, the quality of the GPS signal is quite weak, moreover, in the regions where key military infrastructure facilities are located, signal jamming is enforced. Besides, the common Russian satellite communication system GLONASS does not always ensure seamless communication. During approbation of the proposed approach to robot local navigation, an UAV-aided MRP localization algorithm was successfully tested. Upon the accuracy estimate of this algorithm a reverse dependency was revealed between an absolute MRP localization error and the number of UAVs in action. Thereby the decrease of positive impact was shown by addition of every extra UAV: when using 6 UAVs instead of a minimum set of 3 UAVs, an average accuracy gain was $67.4 \%$, whereas in the case of 12 UAVs instead of $6-25.6 \%$. Besides, it was shown, that with the addition of UAVs in action also the area increases, within which the group of MRP can be localized: from $1.04 \mathrm{~km}^{2}$ to $2.49 \mathrm{~km}^{2}$ when using 3 and 12 UAVs, respectively.
Within this research, MRP navigation accuracy also was estimated using the developed approach. In terms of the respective experiment, downward dependency was revealed between the averaged value of MRP position deflection and the number of available UAVs. The highest value of this metric was revealed in the case with 6 UAVs, equal to $57.12 \mathrm{~m}$, whereas the lowest one for $20 \mathrm{UAVs}$, equal to $16.38 \mathrm{~m}$. Therefore, we can conclude, that navigation accuracy in terms of the developed approach increases significantly with a greater number of UAVs in action and suffices to implement the developed approach in the domain of agricultural robotics.

Further research will focus on optimizing the solution presented, including determination of the best UAV constellation than the distribution of devices along the vertices of a regular polygon, as well as alternative expressions for assessing the MRP expected location.

\section{REFERENCES}

[1] V. I. Nabokov and E. A. Skvortsov, "Classification and features of robotics in agriculture," Agrarian Bulletin of the Urals, vol. 2, no. $156,2017$.

[2] M. Negrete, J. Savage and L. Contreras-toledo, "A motion-planning system for a domestic servicerobot," SPIIRAS Proceedings, vol. 60, no. 5, pp. 5-38, 2018.

[3] S. Hong, M. Li, M. Liao and P. van Beek, "Real-time mobile robot navigation based on stereo vision and low-cost GPS," Electronic Imaging, vol. 44, pp. 10-15, 2017.

[4] J. M. Bengochea-Guevara, J. Conesa-Muñoz, D. Andújar and A. Ribeiro, "Merge fuzzy visual servoing and GPS-based planning to obtain a proper navigation behavior for a small crop-inspection robot," Sensors, vol. 16, no. 3, p. 276, 2016.

[5] A. Denisov and A. Saveliev, "Comparative analysis of wireless data exchange technologies for IoT-system realization," Information technologies and systems, p. 422-429, 2018.

[6] T. Rabie and S. Suleiman, "A novel wireless mesh network for indoor robotic navigation," in 2016 th International Conference on Electronic Devices, Systems and Applications (ICEDSA), IEEE, p. $1-4,2016$.

[7] K. Hattori, N. Tatebe and T. Kagawa, "Deployment of wireless mesh network using RSSI-based swarm robots," Artificial Life And Robotics, vol. 21, no. 4, p. 434-442, 2016

[8] A. Lavric and V. Popa, "Internet of things and LoRa ${ }^{\mathrm{TM}}$ low-power wide-area networks: a survey," in 2017 International Symposium on Signals, Circuits and Systems (ISSCS), IEEE, p. 1-5, 2017.

[9] C. H. Barriquello, D. P. Bernardon, L. N. Canha, F. E. S. e Silva, and D. S. Porto, "Performance assessment of a low power wide area network in rural smart grids," in 2017 52nd International Universities Power Engineering Conference, IEEE., p. 1-4, 2017.

[10] LoRa Alliance Available: https://lora-alliance.org (accessed 15.05.2020)

[11] A.V. Denisov, "Development of a recommender system for parameter calculation in wireless network of sensor devices," MOIT, vol. 7 , no. 4,2019

[12] H. Kremo, T. FarrelE and J. Tallon, "A method to enhance ranging resolution for localization of LoRa sensors," in 2017 IEEE 28th Annual International Symposium on Personal, Indoor, and Mobile Radio Communications (PIMRC), IEEE, p. 1-6, 2017.

[13] Tello, Available: htps:/www.ryzerobotics.com/tello (accessed: 30.04.2020).

[14] N. Pavliuk, I. Kharkov, E. Zimuldinova and V. Saprychev, "Development of Multipurpose Mobile Platform with a Modular Structure," in Proceedings of 14th International Conference on Electromechanics and Robotics "Zavalishin's Readings", Springer, Singapore, p. 137-147, 2020.

[15] N. A. Pavliuk, P. A. Smirnov and A. M. Kovalev, "Constructional and architectural solutions for service mobile platform with pluggable modules," Izvestiya TulGU. Technical science, vol. 10, pp. 181-193, 2019.

[16] GIS Satellite Available: https://www.geoscan.aero/ru/software/sputnik/gis (accessed: 30.04.2020). 